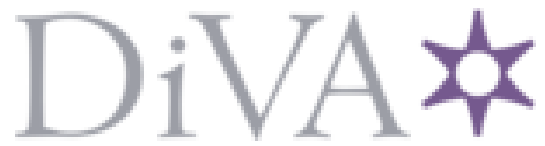

http://www.diva-portal.org

Preprint

This is the submitted version of a paper presented at Solid-State Sensors, Actuators and Microsystems (TRANSDUCERS), 2017 19th International Conference on.

Citation for the original published paper:

Schröder, S. (2017)

A SINGLE WIRE LARGE-AREA FILAMENT EMITTER FOR SPECTROSCOPIC ETHANOL GAS SENSING FABRICATED USING A WIRE BONDING TOOL. In:

N.B. When citing this work, cite the original published paper.

Permanent link to this version:

http://urn.kb.se/resolve?urn=urn:nbn:se:kth:diva-214450 


\title{
A SINGLE WIRE LARGE-AREA FILAMENT EMITTER FOR SPECTROSCOPIC ETHANOL GAS SENSING FABRICATED USING A WIRE BONDING TOOL
}

\author{
Stephan Schröder ${ }^{1,2}$, Henrik Rödjegård ${ }^{2}$, Göran Stemme ${ }^{1}$ and Frank Niklaus ${ }^{1}$ \\ ${ }^{1}$ Department of Micro and Nanosystems, KTH Royal Institute of Technology, Stockholm, SWEDEN \\ ${ }^{2}$ SenseAir AB, Delsbo, SWEDEN
}

\begin{abstract}
Non-dispersive infrared (NDIR) gas spectroscopy is a highly accurate optical gas sensing technology, which has been implemented in various industrial applications. However NDIR systems remain too expensive for many consumer and automotive applications. The cost of the infrared (IR) emitter component is a substantial part of the total system cost. In this paper we report of a single filament IR emitter that is fabricated using wire bonding technology. Our fabrication approach offers the prospect of a fully automated assembly by means of utilizing a wire bonding tool to integrate the single filament to the MEMS structured silicon substrate. An applicationspecific wire bond trajectory enables the mechanical attachment of the filament to form the meander-shaped emitter with a total area of $1 \mathrm{~mm}^{2}$. The fabricated IR emitter utilizes a Kanthal $(\mathrm{FeCrAl})$ filament with very high thermal stability and excellent emitting properties under atmospheric conditions. The packaged IR emitter has been characterized using Fourier transform infrared (FTIR) spectroscopy to study the emitted IR spectrum with respect to the requirements of NDIR systems.
\end{abstract}

\section{KEYWORDS}

NDIR, non-dispersive infrared gas sensing, ethanol gas sensing, breath alcohol sensing, infrared emitter, wire bonding, non-bondable wire materials, integration platform, Kanthal filament.

\section{INTRODUCTION}

NDIR gas sensor systems provide highly accurate quantitative and qualitative real-time measurements of very-low concentrations of gases, such as carbon dioxide, methane, ethanol and other trace gases that show a wavelength specific absorption in the infrared spectrum, which is the so called finger print region $[1,2,3]$. These gas sensors have been implemented in various industrial applications, such as processing and agricultural industry and in heating, ventilation and air conditioning (HVAC) systems, to monitor carbon dioxide concentrations. Consequently NDIR systems are a proven, reliable and powerful non-contact gas sensing technology. Utilizing NDIR systems to monitor elevated breath alcohol concentration (BrAC) is an important emerging application in work environments with security-relevant concerns, in hazardous working environments and ultimately for integration in miniaturized breath alcohol ignition interlock devices (BAIID) for automotive vehicles to prevent alcohol-intoxicated persons from vehicle usage [4]. Currently available ethanol sensors are based on either the conductometric semiconductor metal oxide principle or on direct alcohol fuel cells (DAFC) [5]. All these sensors principles suffer from low-accuracy,

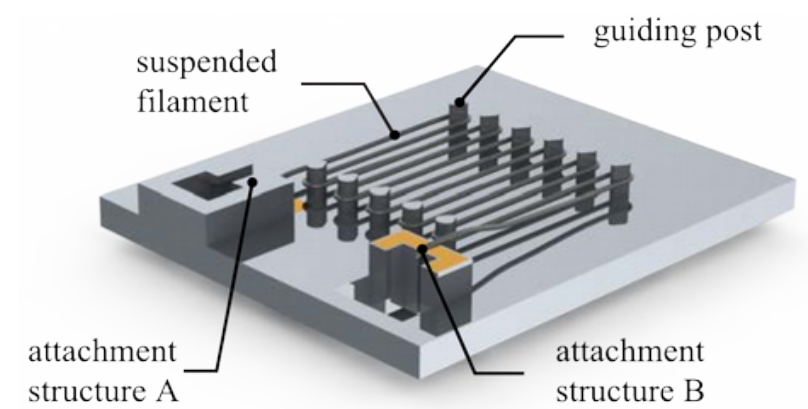

Figure 1: 3D model of the infrared emitter based on a single wire large-area filament. The suspended filament is mechanically fixated using attachment structures and two rows of out-of-plane guiding posts.

frequent maintenance and the need for extensive exhalation volumes, making these gas sensing systems unattractive for automotive applications $[6,7]$. Thus, there is a strong need for ethanol sensors, such as NDIR ethanol sensors, that overcome current obstacles to monitor effectively and precisely elevated BrAC levels, while being entirely maintenance-free, cost-efficient, smallsized and reliable.

Conventional NDIR sensor systems are distinguished by their simple structure, consisting of an IR emitter, in general a low-cost incandescent light bulb to emit IR radiation into a multipath spectroscopic absorption cell, i.e. the White cell, where the IR beam is directed multiple times back and forth and is partially absorbed by the target gas. Next, the intensity of the IR radiation is measured by a wavelength selective IR detector and is related to the concentration of the target gas in the White cell. The characteristics of the IR emitter are of crucial importance and determine in particular the performance of the NDIR sensor system. While traditional NDIR systems for carbon dioxide sensing make use of incandescent light bulbs as IR emitter, the usage of such cost-effective components is per se not a suitable IR emitter for ethanol sensing, as the transparency of the glas envelop of the light bulb drops significantly for wavelengths beyond $4.5 \mu \mathrm{m}$. Detection of elevated BrAC levels based on NDIR technology utilizes exhaled carbon dioxide as a reference gas. Therefore a broadband IR emitter is required that radiates within the middle and far IR wavelength spectrum as ethanol exhibits a utilizable absorption mode at a wavelength of $9.5 \mu \mathrm{m}$ and carbon dioxide an absorption mode at a wavelength of $4.3 \mu \mathrm{m}$.

Current solid-state and MEMS-based IR emitters suffer from comparably high fabrication costs, as exotic semiconductor or silicon-on-insulator (SOI) substrates are utilized [8]. Platinum as the emitting material is frequently proposed but suffers from low-emissivity in the IR wavelength range, and thermally induced mechanical stresses due to high operation temperatures can negatively 


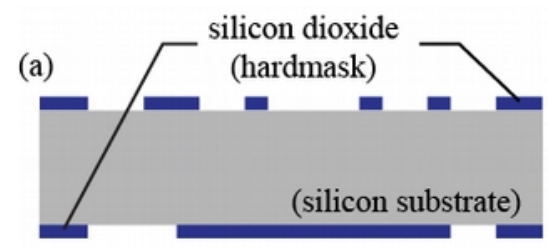

2nd top side dry etch

(d) sequence (isotropic)

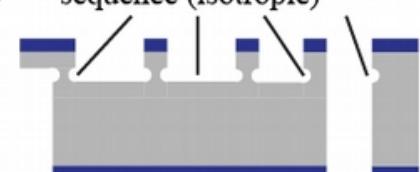

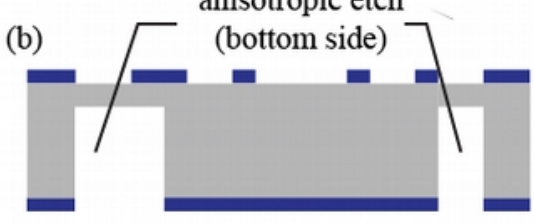

3rd top side dry etch

(e) sequence (anisotropic)

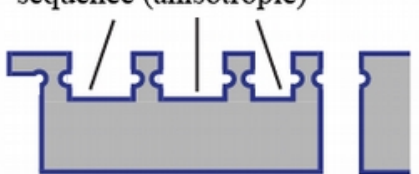

1st top side dry etch sequence (anisotropic)
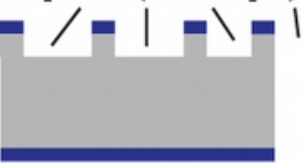

(f)

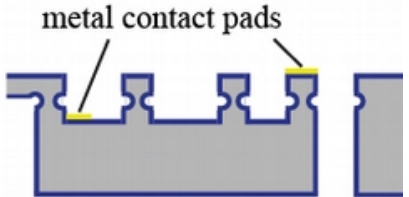

Figure 2: Fabrication scheme of the emitter substrate. (a) A silicon substrate is oxidized, patterned using lithography and dry etched to from the silicon dioxide hard mask. (b) Anisotropic dry etching of the bottom side of the substrate. (e-c) Multiple dry etching sequence consisting of an anisotropic, isotropic and anisotropic step forming guiding posts and attachment structures. (e-f) Removal of the oxide mask and re-oxidation and final formation of metal contact pads.

affect the reliability of the IR emitter. As a consequence, existing IR emitters are not attractive devices for low-cost ethanol NDIR spectroscopy systems.

Wire bonding is a very mature, effective and reliable electrical interconnection technology, which has been proposed for multiple innovative MEMS packaging and heterogeneous integration approaches $[9,10]$. Recently we have reported of an integration platform for filamentbased emitters, that necessitate multiple filament integration process steps, resulting in an elaborate fabrication sequence [11]. Here we report on a rapid and more cost-effective fabrication for IR emitters by integrating a single wire filament utilizing an automated wire bonder.

\section{EMITTER DESIGN AND FABRICATION}

The $3 \mathrm{D}$ model of the proposed infrared emitter is illustrated in figure 1. The substrate consist of two attachment structures enabling the mechanical fixation of the filament. Attachment structure A (figure 3a) consists of a vertical trench having a depth of $40 \mu \mathrm{m}$ and a width of $90 \mu \mathrm{m}$ and opens up a buried cavity that allows the mechanical fixation of the free air ball (FAB) at the beginning of the filament. Attachment structure B (figure $3 \mathrm{c} \& 3 \mathrm{~d}$ ) consists of a tapered vertical trench having a width between $18 \mu \mathrm{m}$ and $28 \mu \mathrm{m}$ to fixate the end of the filament. Two rows of guiding posts with a distance of $1 \mathrm{~mm}$ facilitate the filament wire placement to form the meander-shaped emitter. The pitch of the guiding posts within one row is $200 \mu \mathrm{m}$ and each of the guiding posts possesses a recess to ensure that the filament is suspended at a defined height. This prevents direct physical contact of the filament to the substrate surface. The pitch between each meander loop of the filament wire is about $100 \mu \mathrm{m}$ and the integrated filament with a total length of $15.8 \mathrm{~mm}$ forms a large emitting area of $1 \mathrm{~mm}^{2}$. Next to the attachment structure A and on top of the attachment structure B are metal pads enabling the formation of electrical contacts between the emitter filament using conventional stud bumping. Fabrication of the infrared emitter is accomplished in a sequential work flow: First the structuring of the emitter substrate using conventional MEMS fabrication processes and second the integration and contact formation of the emitter filament.

\section{Substrate fabrication}

Emitter devices are fabricated using a $100 \mathrm{~mm}$ diameter double-side polished silicon wafer with a thickness of $300 \mu \mathrm{m}$. A thermal wet oxidation grows a $1 \mu \mathrm{m}$ thick silicon dioxide layer. Patterning of the top and bottom side of the substrate using lithography and dry etching of silicon dioxide defines the hard mask for silicon etching (figure $2 \mathrm{a}$ ). An anisotropic silicon dry etch of the bottom side opening up the $260 \mu \mathrm{m}$ deep and $250 \mu \mathrm{m}$ wide buried recesses (figure $2 \mathrm{~b}$ ). The dry etch process on the top side of the substrate consists of a sequence of three silicon etching steps for finalizing the attachment structures and the guiding posts, simultaneously. First an anisotropic etch is performed to form the vertical trenches and opens up the buried recess of the attachment structures A, generating the tapered trench of attachment structures B for fixating the end of the filament and the protruding heads of the guiding posts (figure 2c). Next, an isotropic etch generates the shape of the recesses of the guiding posts for the filament placement (figure 2d) and a final anisotropic etch sequence defines the final out-of plane height of the guiding posts. The silicon dioxide hard mask is stripped in a wet etching process using hydrofluoric (HF) acid. Thermal re-oxidation grows another silicon dioxide layer with a thickness of $500 \mathrm{~nm}$ to electrically insulate the attachment structures and the guiding posts (figure 2e). Titanium and gold is deposited on the top side of the wafer for the metal contact pad formation. Lithographic patterning using a spray-coated resist and wet etching of titanium and gold finalizes the metal contact pad formation (figure $2 \mathrm{f}$ ).

\section{Filament placement}

For the filament integration the fabricated substrate is diced in chips, mounted on a carrier substrate and placed on the heater plate of the wire bonder. In this work we have used resistive heating wires with a diameter of $25 \mu \mathrm{m}$ made of Kanthal (FeCrAl) alloy, which can be operated at temperatures of up to $1200{ }^{\circ} \mathrm{C}$, offers superior aging and emission characteristics and is very suitable for operation under atmospheric conditions. Placement of the filament and mechanical attachment are performed using an automated wire bonder, type ESEC 3100 plus, Besi, 
(a)

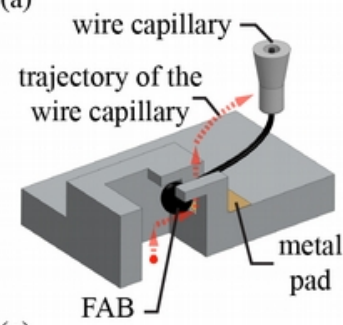

(c)

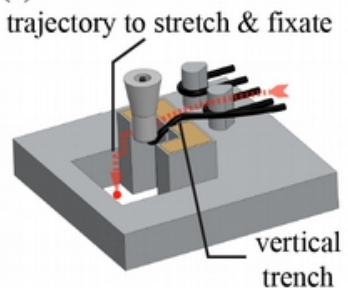

(b)

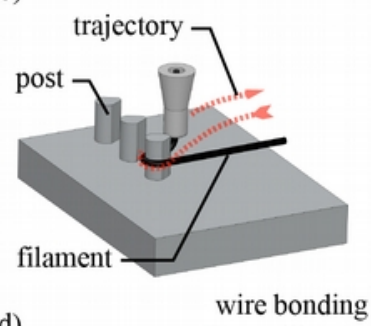

(d)

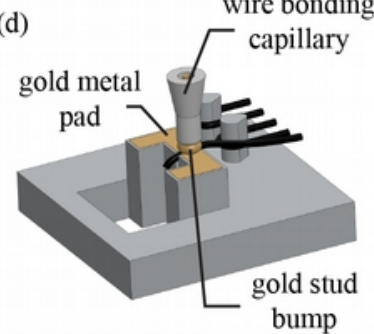

Figure 3: Illustration of the filament placement no the emitter substrate and the contact formation using a wire bonding tool. (a) The trajectory (red dashed) of the wire capillary guides the free air ball into the attachment structure A. (b) The wire capillary places sequentially the filament around the guiding posts, thereby forming the meander-shaped emitting zone. (c) After the filament placement the wire capillary stretches the filament and forces it into the vertical trench to establish a mechanical fixation to the attachment structure $A$. (d) Electrical contact formation by bonding a gold stud on the metal pads of each attachment structure, thereby embedding the integrated filament.

Switzerland. Initially a FAB with a diameter of $110 \mu \mathrm{m}$ is formed by an electronic flame off (EFO) in an inert gas atmosphere. The FAB is centered underneath the wire capillary that has a significantly smaller outer diameter of $85 \mu \mathrm{m}$ compared to the FAB. Next, the wire capillary is lowered into the bottom of attachment structure A. Figure 3a illustrates details of attachment structure A and indicates the executed trajectory of the wire capillary highlighted in dashed red. At the bottom of attachment structure A, a ball bond without mechanical deformation of the FAB is performed to define the origin of the application-specific trajectory of the wire capillary. The wire capillary places the FAB into the buried recess and moves vertically out of the attachment structure, thereby fixating the FAB into the attachment structure A. Next, the wire capillary moves towards the first guiding post and performs a circular move to wind the filament around the guiding post into the defined recess of the post (figure $3 b)$. Sequential alternating of the latter trajectory sections completes the filament placement around the remaining guiding posts and the wire capillary is centered above the attachment structure B. A stitch bond stretches the filament and presses gradually the filament into the tapered trench until a press-fit attachment is established. High bond forces and ultrasonic energy truncate the filament and finalizes the filament integration process (figure 3c). Electrical contact formation between the filament and the substrate is performed by using conventional stud bumping technology. Therefore, a gold stud bump with a diameter of $60 \mu \mathrm{m}$ is centered above the metal contact pad and the filament. The gold ball is

bonded to the metal pad and thereby enclosing the filament in the gold ball. By this enclosure oxidation of the filament surface at the gold to filament contact interface is avoided (figure 3d). After the contact formation a burn-in is performed to oxidize the filament surface in air atmosphere at about $40 \%$ humidity. Therefore a current of $200 \mathrm{~mA}$ is forced for $1 \mathrm{~min}$ through the emitter.

\section{RESULTS AND DISCUSSION}

The fabricated IR emitter is shown in figure 4 after the filament integration process. Both ends of the filament are mechanically fixated to the attachment structures A and $\mathrm{B}$ and the filament is winded around 11 guiding posts. The application-specific trajectory allows a very precise placement of the filament, while a constant strain of the filament during the placement process is maintained to avoid any physical contact to the substrate surface. The close-up in figure 4 illustrates the winded filament around one of the guiding posts that utilizes the recess for the defined out-of-plane placement. The filament of the IR emitter device has a length of $15.8 \mathrm{~mm}$ and is covering an area of $1 \mathrm{~mm}^{2}$. The pitch between each filament loop is about $100 \mu \mathrm{m}$.

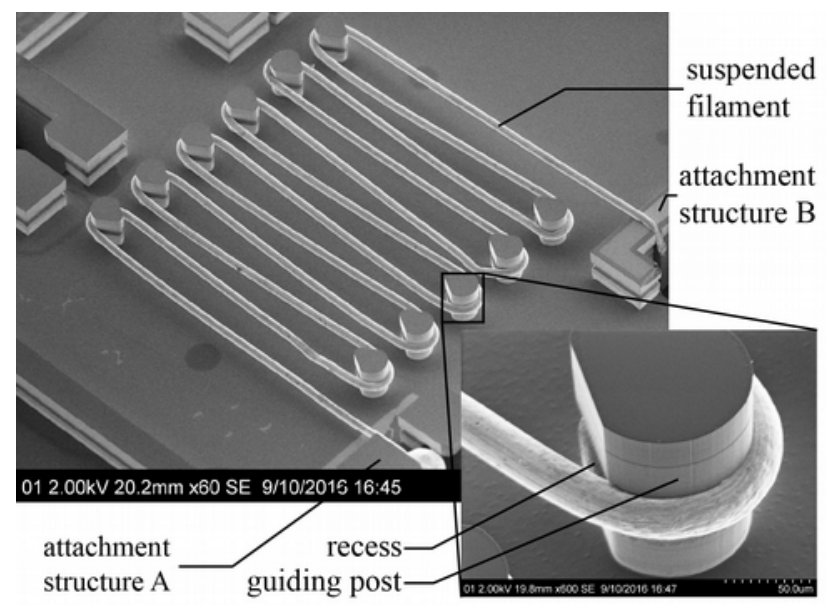

Figure 4: SEM image of the fabricated meander-shaped IR emitter. The filament is mechanically fixated at its ends to the attachment structures $A$ and B. Guiding posts are utilized to suspend the filament in the meander-shape above the substrate surface, forming an emitting zone of $1 \mathrm{~mm}^{2}$. The close-up illustrates details of the guiding post and its recess that enables the stable fixation of the filament at a defined height above the substrate surface.

Figure 5 illustrates details of the attachment structures A (left) and B (right) after the filament placement. As the diameter of the FAB of about $110 \mu \mathrm{m}$ exceeds the width of the vertical trench of $90 \mu \mathrm{m}$, the FAB is fastened reliably to the buried recess and the filament is guided out of the vertical trench. The image to the right in figure 5 shows the end of the filament pressed into the tapered vertical trench at about $22 \mu \mathrm{m}$ width, thereby establishing a press-fit like attachment. The end of the truncated Kanthal filament is deformed from high bond forces and ultrasonic energy. Characterization of the fabricated IR emitter is performed using Fourier transform infrared (FTIR) spectroscopy. Since NDIR spectroscopy for BrAC sensing utilizes carbon dioxide as a reference gas an IR 

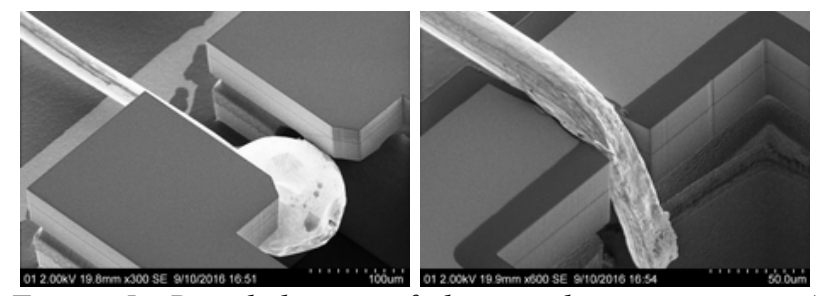

Figure 5: Detailed view of the attachment structure $A$ with the attached FAB in the buried recess (left) and close-up of attachment structure B (right). The end of the filament is forced into the tapered vertical trench where a press-fit fixation is established.

emission spectrum of the IR emitter between $1 \mu \mathrm{m}$ and $15 \mu \mathrm{m}$ has been studied. Ethanol shows a relatively strong IR absorption at $9.5 \mu \mathrm{m}$, whereas no other substantial IR absorption from other relevant gases occur. Carbon dioxide shows a strong absorption of infrared radiation at $4.3 \mu \mathrm{m}$. Figure 6 shows the measured emission spectrum of the meander-shaped IR emitter, when the device is driven by a constant current of $200 \mathrm{~mA}$. The emission spectrum shows a relatively good correlation to a black body radiator with respect to the emissivity of 0.7 for Kanthal filaments. However the constant current results in substrate heating and thus the IR spectrum differs from a black body radiator at longer wavelengths. The minima in the emission spectrum at $2.8 \mu \mathrm{m}$ and between $6.1 \mu \mathrm{m}$ and $6.8 \mu \mathrm{m}$ are based on the infrared absorption of water, while the dip at $4.3 \mu \mathrm{m}$ is caused by IR absorption of carbon dioxide.

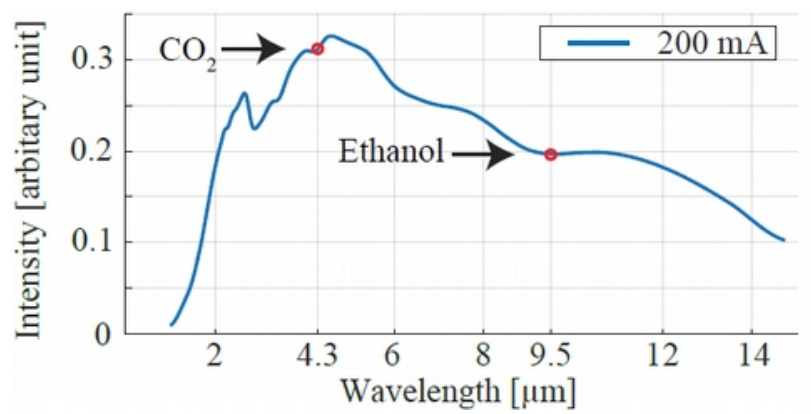

Figure 6: Graph of the measured IR emission spectrum of the fabricated large-area IR emitter in the wavelength range between $1 \mu \mathrm{m}$ and $15 \mu \mathrm{m}$ versus the intensity. Local minima at about $2.8 \mu \mathrm{m}, 4.3 \mu \mathrm{m}$ and between $6.1 \mu \mathrm{m}$ and $6.8 \mu \mathrm{m}$ are caused by the IR absorption of water and carbon dioxide. The absorption wavelength of interest for NDIR gas sensing of carbon dioxide and ethanol at $4.3 \mu \mathrm{m}$ and $9.5 \mu \mathrm{m}$ are labeled.

\section{CONCLUSION}

We have demonstrated the successful fabrication of a single filament IR emitter that is suitable for NDIR ethanol gas sensing using an integration platform for nonbondable wire materials with a fully automated wire bonding tool. The utilized Kanthal emitter filament is mechanically attached on a structured silicon substrate and placed in a meander shape forming a large emitting area of $1 \mathrm{~mm}^{2}$. The emitter has been successfully operated and the radiated emission spectrum of the IR emitter has been studied using FTIR spectroscopy. The relevant spectrum between a wavelength range of $1 \mu \mathrm{m}$ and $15 \mu \mathrm{m}$ shows a suitable IR spectrum for NDIR gas sensing of carbon dioxide and ethanol at $4.3 \mu \mathrm{m}$ and $9.5 \mu \mathrm{m}$.

\section{ACKNOWLEDGEMENTS}

Support from the European Commission through the ERC Starting Grant (M\&M's, 277879), the Swedish Research Council (621-2011-4437) and Vinnova (201500402) is gratefully acknowledged.

\section{REFERENCES}

[1] J. Hodgkinson and R. P. Tatam, "Optical gas sensing: a review," Measurement Science and Technology, vol. 24, no. 1, p. 012004, nov 2012. http://dx.doi.org/10.1088/0957-0233/24/1/012004

[2] X. Liu, S. Cheng, H. Liu, S. Hu, D. Zhang, and H. Ning, "A Survey on Gas Sensing Technology," Sensors, vol. 12, no. 12, pp. 9635-9665, jul 2012. http://dx.doi.org/10.3390/s120709635

[3] Rödjegård, H. "IR-sensors for measurement of greenhouse gases." Proceedings of Micronano Systems Workshop MSW. 2010.

[4] J. Ljungblad, B. Hök, and M. Ekström, "Critical performance of a new breath alcohol analyzer for screening applications," in Intelligent Sensors, Sensor Networks and Information Processing (ISSNIP), 2014 IEEE Ninth International Conference on. IEEE, 2014, pp. 1-4.

[5] Hok, Bertil, et al. "Breath analyzer for alcolocks and screening devices." IEEE Sensors Journal 10.1 (2010): 10-15.

[6] A. Jonsson, B. Hök, L. Andersson, and G. Hedenstierna, "Methodology investigation of expirograms for enabling contact free breath alcohol analysis," Journal of breath research, vol. 3, no. 3, p. 036002, 2009.

[7] C. Bax, O. E. Kärki et al., "Alcohol interlock implementation in the European union: feasibility study. final report of the European research project," 2002.

[8] Dinh, Trieu-Vuong, et al. "A review on nondispersive infrared gas sensors: Improvement of sensor detection limit and interference correction." Sensors and Actuators B: Chemical 231 (2016): 529538.

[9] A. C. Fischer, H. Gradin, S. Schröder, S. Braun, G. Stemme, W. van der Wijngaart, and F. Niklaus, "Wire-bonder-assisted integration of non-bondable SMA wires into MEMS substrates," J. Micromech. Microeng., vol. 22, no. 5, p. 055025, apr 2012.

[10] Fischer, Andreas C., et al. "Unconventional applications of wire bonding create opportunities for microsystem integration." Journal of Micromechanics and Microengineering 23.8 (2013): 083001.

[11] Schröder, Stephan, et al. "Fabrication of an infrared emitter using a generic integration platform based on wire bonding." JMM 26.11 (2016): 115010.

\section{CONTACT}

S. Schröder, tel: +46702555337 ; stephan.schroder@ee.kth.se; stephan.schroder@senseair.se. 